\title{
Crystal Structure of 2-Trifluoromethyl-4'-dimethylaminoazobenzene
}

\author{
Chizuru Sasaki, ${ }^{*}$ Soh-ichi Kitoh, ${ }^{*}$ Kazuya Yamada, ${ }^{*}$ Ko-Ki Kunimoto, ${ }^{* \dagger}$ Shiro Maeda, ** \\ Akio KuWAE, *** and Kazuhiko HaNaI*** \\ *Department of Chemistry and Chemical Engineering, Faculty of Engineering, Kanazawa University, \\ Kakuma-machi, Kanazawa 920-1192, Japan \\ **Department of Applied Chemistry and Biotechnology, Faculty of Engineering, Fukui University, \\ Bunkyo, Fukui 910-8507, Japan \\ ***Graduate School of Natural Sciences, Nagoya City University, Mizuho-ku, Nagoya 467-8501, Japan
}

\begin{abstract}
Crystals of 2-trifluoromethyl-4'-dimethylaminoazobenzene (2-TFMDAB) are orthorhombic, space group Pbca with $a=$ $15.625(4), b=23.028(6), c=7.685(2) \AA$, and $Z=8$. The structure was solved by direct methods (SIR88) and refined to a final $R$ value of 0.049 for 2536 reflections $(I>0.80 \sigma(I))$. 2-TFMDAB has a trans geometry about the azo linkage. The bond lengths and angles are similar to those of other azobenzene compounds. The abnormal UV-vis absorption spectrum in an acidic solution can be explained by the existence of the bulky and electronegative $\mathrm{CF}_{3}$ group at the 2-position, which prevents protonation at the $\mathrm{N} \beta$ atom of the azo group in an acidic solution.
\end{abstract}

(Received October 2, 2002; Accepted February 3, 2003; Published on Web April 30, 2003)

4'-Dimethylaminoazobenzene dyes are used as acid-base indicators because of a discernible color change upon a change of the $\mathrm{pH}$. Typically, an aqueous solution of this dye group is colored yellow at neutral-to-alkaline $\mathrm{pH}$, and turns orange-red when the $\mathrm{pH}$ is lowered to below the color-change interval. This color change has been ascribed to a structure change from the neutral azo form (I) to the protonated azo form (IIa), which has a resonance structure of the quinoid form (IIb), as shown in Fig. $1 .{ }^{1}$ In acidic solution, the protonated azo form (II) is in equilibrium with the colorless ammonium form (III). The chemical species (I), (II) and (III) are associated with absorptions at around 460, 500 and $320 \mathrm{~nm}$, respectively, in the UV-vis absorption spectra. We have investigated the resonance Raman spectra of aqueous solutions of 4'dimethylaminoazobenzene dyes, and characterized the Raman

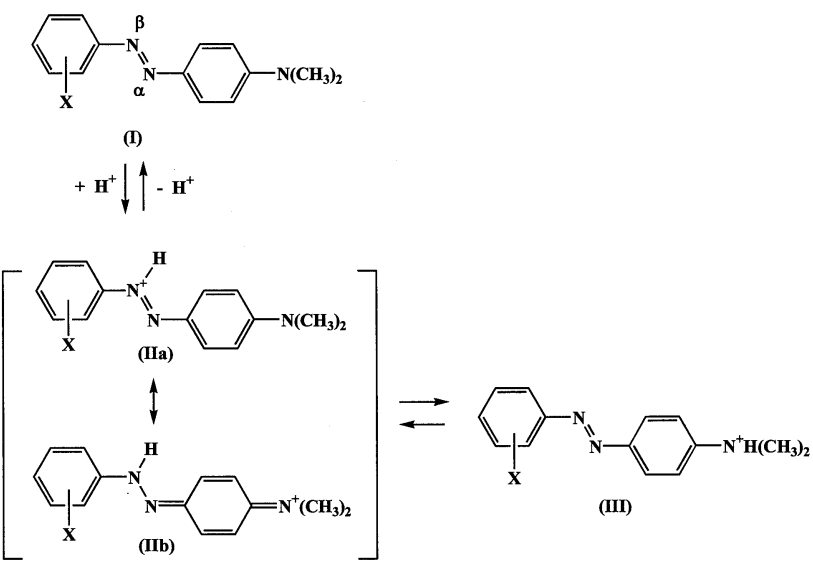

Fig. 1 Protonation equilibrium of $4^{\prime}$-dimethylazobenzene dye. The substituent $\mathrm{X}$ is $4-\mathrm{SO}_{3}^{-}$for methyl orange, 2- $\mathrm{COOH}$ for methyl red and 2-CF3 for 2-TFMDAB.

$\doteqdot$ To whom correspondence should be addressed. bands which originated from molecular species (I), (II) and (III). ${ }^{2-4}$ During the course of a study on the complex formation of azo dyes with peptides, we prepared 2-trifluoromethyl-4' dimethylaminoazobenzene (2-TFMDAB). Unlike other 4'dimethylaminoazobenzene dyes, this compound exhibits a color change from yellow to colorless upon going from neutral to acidic $\mathrm{pH}$. In this work, we undertook the X-ray analysis of 2TFMDAB in order to examine the structural features that

Table 1 Crystal and experimental data

Formula: $\mathrm{C}_{15} \mathrm{H}_{14} \mathrm{~N}_{3} \mathrm{~F}_{3}$

Formula weight: 293.29

Crystal color, habit: orange-red, plates

Crystal size: $0.20 \times 0.13 \times 0.03 \mathrm{~mm}$

Crystal system: orthorhombic

Space group: $P$ bca

$Z=8$

Radiation: $\mathrm{Mo} \mathrm{K}_{\alpha}(0.71069 \AA)$

$T=123 \mathrm{~K}$

$2 \theta_{\max }=55.0^{\circ}$

$F\left(\begin{array}{lll}0 & 0 & 0\end{array}\right)=1216.00$

$a=15.625(4) \AA$

$b=23.028(6) \AA$

$c=7.685(2) \AA$

$V=2765(1) \AA^{3}$

$D_{\text {calc }}=1.409 \mathrm{~g} / \mathrm{cm}^{3}$

$\mu=1.14 \mathrm{~cm}^{-1}$

$R, R \mathrm{w}=0.049,0.070$

No. observations $=2536(I>0.80 \sigma(I))$

No. variables $=246$

Goodness of fit $=0.98$

$(\Delta / \sigma)_{\max }=0.000$

$(\Delta \rho)_{\max }=0.33 \mathrm{e}^{-3}$

$(\Delta \rho)_{\min }=-0.36 \mathrm{e}^{-3}$

Measurement: Quantum CCD/Rigaku AFC-7

Program system: teXsan

Structure determination: direct method (SIR88)

Refinement: full-matrix least-squares 
Table 2 Positional parameters of non-hydrogen atoms

\begin{tabular}{crrrl}
\hline Atom & $x$ & $y$ & $z$ & $B_{\mathrm{eq}}\left(\AA^{2}\right)$ \\
\hline $\mathrm{F}(1)$ & $0.2506(1)$ & $0.26046(9)$ & $0.5985(3)$ & $2.74(5)$ \\
$\mathrm{F}(2)$ & $0.1623(1)$ & $0.20898(9)$ & $0.7428(3)$ & $2.76(5)$ \\
$\mathrm{F}(3)$ & $0.2183(2)$ & $0.1755(1)$ & $0.5090(3)$ & $3.65(6)$ \\
$\mathrm{N}(1)$ & $0.2259(2)$ & $0.0956(1)$ & $0.8096(4)$ & $1.76(6)$ \\
$\mathrm{N}(2)$ & $0.2307(2)$ & $0.0440(1)$ & $0.8677(4)$ & $1.65(6)$ \\
$\mathrm{N}(3)$ & $-0.0609(2)$ & $-0.0957(1)$ & $0.8298(4)$ & $2.00(6)$ \\
$\mathrm{C}(1)$ & $0.2352(2)$ & $0.2064(2)$ & $0.6526(5)$ & $2.00(7)$ \\
$\mathrm{C}(2)$ & $0.3087(2)$ & $0.1822(1)$ & $0.7540(5)$ & $1.69(7)$ \\
$\mathrm{C}(3)$ & $0.3838(2)$ & $0.2149(2)$ & $0.7707(5)$ & $1.99(8)$ \\
$\mathrm{C}(4)$ & $0.4511(2)$ & $0.1944(2)$ & $0.8700(5)$ & $2.09(8)$ \\
$\mathrm{C}(5)$ & $0.4442(2)$ & $0.1416(2)$ & $0.9544(5)$ & $2.06(8)$ \\
$\mathrm{C}(6)$ & $0.3715(2)$ & $0.1084(2)$ & $0.9392(5)$ & $1.91(8)$ \\
$\mathrm{C}(7)$ & $0.3036(2)$ & $0.1277(2)$ & $0.8361(5)$ & $1.73(7)$ \\
$\mathrm{C}(8)$ & $0.1561(2)$ & $0.0107(1)$ & $0.8460(4)$ & $1.59(7)$ \\
$\mathrm{C}(9)$ & $0.1569(2)$ & $-0.0446(2)$ & $0.9221(5)$ & $1.79(7)$ \\
$\mathrm{C}(10)$ & $0.0856(2)$ & $-0.0801(2)$ & $0.9161(5)$ & $1.81(7)$ \\
$\mathrm{C}(11)$ & $0.0105(2)$ & $-0.0618(1)$ & $0.8340(4)$ & $1.61(7)$ \\
$\mathrm{C}(12)$ & $0.0106(2)$ & $-0.0063(2)$ & $0.7539(5)$ & $1.85(7)$ \\
$\mathrm{C}(13)$ & $0.0816(2)$ & $0.0290(1)$ & $0.7612(5)$ & $1.79(7)$ \\
$\mathrm{C}(14)$ & $-0.0606(3)$ & $-0.1522(2)$ & $0.9101(7)$ & $2.85(10)$ \\
$\mathrm{C}(15)$ & $-0.1385(3)$ & $-0.0761(2)$ & $0.7425(6)$ & $2.21(8)$ \\
\hline
\end{tabular}

$B_{\text {eq }}=(8 / 3) \pi^{2}\left(U_{11}\left(a a^{*}\right)^{2}+U_{22}\left(b b^{*}\right)^{2}+U_{33}\left(c c^{*}\right)^{2}+2 U_{12} a a^{*} b b^{*} \cos \gamma\right.$ $\left.+2 U_{13} a a^{*} c c^{*} \cos \beta+2 U_{23} b b^{*} c c^{*} \cos \alpha\right)$.

Table 3 Selected bond lengths $(\AA)$, bond angles $\left({ }^{\circ}\right)$ and torsion angles $\left({ }^{\circ}\right)$

\begin{tabular}{llllll}
\hline Atom & Atom & Distance & Atom & Atom & Distance \\
\hline $\mathrm{F}(1)$ & $\mathrm{C}(1)$ & $1.334(4)$ & $\mathrm{F}(2)$ & $\mathrm{C}(1)$ & $1.335(4)$ \\
$\mathrm{F}(3)$ & $\mathrm{C}(1)$ & $1.339(4)$ & $\mathrm{N}(1)$ & $\mathrm{N}(2)$ & $1.271(4)$ \\
$\mathrm{N}(1)$ & $\mathrm{C}(7)$ & $1.437(4)$ & $\mathrm{N}(2)$ & $\mathrm{C}(8)$ & $1.406(4)$ \\
$\mathrm{N}(3)$ & $\mathrm{C}(11)$ & $1.362(4)$ & $\mathrm{N}(3)$ & $\mathrm{C}(14)$ & $1.440(5)$ \\
$\mathrm{N}(3)$ & $\mathrm{C}(15)$ & $1.457(5)$ & $\mathrm{C}(1)$ & $\mathrm{C}(2)$ & $1.495(5)$ \\
\hline
\end{tabular}

\begin{tabular}{llllllll}
\hline Atom & Atom & Atom & Angle & Atom & Atom & Atom & Angle \\
\hline $\mathrm{N}(2)$ & $\mathrm{N}(1)$ & $\mathrm{C}(7)$ & $112.4(3)$ & $\mathrm{N}(1)$ & $\mathrm{N}(2)$ & $\mathrm{C}(8)$ & $114.7(3)$ \\
$\mathrm{C}(11)$ & $\mathrm{N}(3)$ & $\mathrm{C}(14)$ & $120.3(3)$ & $\mathrm{C}(11)$ & $\mathrm{N}(3)$ & $\mathrm{C}(15)$ & $120.9(3)$ \\
$\mathrm{C}(14)$ & $\mathrm{N}(3)$ & $\mathrm{C}(15)$ & $118.7(3)$ & $\mathrm{F}(1)$ & $\mathrm{C}(1)$ & $\mathrm{F}(2)$ & $105.9(3)$ \\
$\mathrm{F}(1)$ & $\mathrm{C}(1)$ & $\mathrm{F}(3)$ & $105.9(3)$ & $\mathrm{F}(1)$ & $\mathrm{C}(1)$ & $\mathrm{C}(2)$ & $111.9(3)$ \\
$\mathrm{F}(2)$ & $\mathrm{C}(1)$ & $\mathrm{F}(3)$ & $106.5(3)$ & $\mathrm{F}(2)$ & $\mathrm{C}(1)$ & $\mathrm{C}(2)$ & $113.6(3)$ \\
$\mathrm{F}(3)$ & $\mathrm{C}(1)$ & $\mathrm{C}(2)$ & $112.5(3)$ & $\mathrm{C}(1)$ & $\mathrm{C}(2)$ & $\mathrm{C}(3)$ & $119.4(3)$ \\
$\mathrm{C}(1)$ & $\mathrm{C}(2)$ & $\mathrm{C}(7)$ & $121.5(3)$ & $\mathrm{N}(1)$ & $\mathrm{C}(7)$ & $\mathrm{C}(2)$ & $116.4(3)$ \\
$\mathrm{N}(1)$ & $\mathrm{C}(7)$ & $\mathrm{C}(6)$ & $123.9(3)$ & $\mathrm{N}(2)$ & $\mathrm{C}(8)$ & $\mathrm{C}(9)$ & $116.0(3)$ \\
$\mathrm{N}(2)$ & $\mathrm{C}(8)$ & $\mathrm{C}(13)$ & $125.6(3)$ & $\mathrm{N}(3)$ & $\mathrm{C}(11)$ & $\mathrm{C}(10)$ & $121.7(3)$ \\
$\mathrm{N}(3)$ & $\mathrm{C}(11)$ & $\mathrm{C}(12)$ & $120.5(3)$ & & & & \\
\hline
\end{tabular}

\begin{tabular}{llllrllllr}
\hline Atom & Atom & Atom & Atom & \multicolumn{1}{c}{ Angle } & Atom & Atom & Atom & Atom & \multicolumn{1}{c}{ Angle } \\
\hline $\mathrm{N}(1)$ & $\mathrm{N}(2)$ & $\mathrm{C}(8)$ & $\mathrm{C}(9)$ & $-174.6(3)$ & $\mathrm{N}(1)$ & $\mathrm{N}(2)$ & $\mathrm{C}(8)$ & $\mathrm{C}(13)$ & $3.0(5)$ \\
$\mathrm{N}(1)$ & $\mathrm{C}(7)$ & $\mathrm{C}(2)$ & $\mathrm{C}(1)$ & $3.1(5)$ & $\mathrm{N}(1)$ & $\mathrm{C}(7)$ & $\mathrm{C}(2)$ & $\mathrm{C}(3)$ & $-178.1(3)$ \\
$\mathrm{N}(1)$ & $\mathrm{C}(7)$ & $\mathrm{C}(6)$ & $\mathrm{C}(5)$ & $178.7(3)$ & $\mathrm{N}(2)$ & $\mathrm{N}(1)$ & $\mathrm{C}(7)$ & $\mathrm{C}(2)$ & $171.1(3)$ \\
$\mathrm{N}(2)$ & $\mathrm{N}(1)$ & $\mathrm{C}(7)$ & $\mathrm{C}(6)$ & $-10.1(5)$ & $\mathrm{N}(2)$ & $\mathrm{C}(8)$ & $\mathrm{C}(9)$ & $\mathrm{C}(10)$ & $177.0(3)$ \\
$\mathrm{N}(2)$ & $\mathrm{C}(8)$ & $\mathrm{C}(13)$ & $\mathrm{C}(12)$ & $-177.1(3)$ & $\mathrm{N}(3)$ & $\mathrm{C}(11)$ & $\mathrm{C}(10)$ & $\mathrm{C}(9)$ & $-178.6(3)$ \\
$\mathrm{N}(3)$ & $\mathrm{C}(11)$ & $\mathrm{C}(12)$ & $\mathrm{C}(13)$ & $178.3(3)$ & $\mathrm{C}(1)$ & $\mathrm{C}(2)$ & $\mathrm{C}(3)$ & $\mathrm{C}(4)$ & $177.2(3)$ \\
$\mathrm{C}(1)$ & $\mathrm{C}(2)$ & $\mathrm{C}(7)$ & $\mathrm{C}(6)$ & $-175.7(3)$ & $\mathrm{C}(7)$ & $\mathrm{N}(1)$ & $\mathrm{N}(2)$ & $\mathrm{C}(8)$ & $179.6(3)$ \\
$\mathrm{C}(10)$ & $\mathrm{C}(11)$ & $\mathrm{N}(3)$ & $\mathrm{C}(14)$ & $-0.3(5)$ & $\mathrm{C}(10)$ & $\mathrm{C}(11)$ & $\mathrm{N}(3)$ & $\mathrm{C}(15)$ & $-179.5(3)$ \\
$\mathrm{C}(12)$ & $\mathrm{C}(11)$ & $\mathrm{N}(3)$ & $\mathrm{C}(14)$ & $179.5(4)$ & $\mathrm{C}(12)$ & $\mathrm{C}(11)$ & $\mathrm{N}(3)$ & $\mathrm{C}(15)$ & $0.3(5)$ \\
\hline
\end{tabular}

Estimated standard deviations in the least-significant fugure are given in parentheses.

account for the abnormal color change.

2-TFMDAB was prepared by the diazotization of 2aminobenzotrifluoride with sodium nitrite, followed by coupling with $N$-dimethylaniline. The crude product was purified through recrystallization from an ethanol solution. Orange-red plate crystals suitable for X-ray diffraction analysis were obtained by the slow evaporation of an aqueous ethanol solution at room temperature (m.p. $105-106^{\circ} \mathrm{C}$ ).

All of the non-hydrogen atoms were refined anisotropically. The positions of the hydrogen atoms were determined from a difference Fourier map and were refined isotropically. The crystal and experimental data are listed in Table 1 . The final

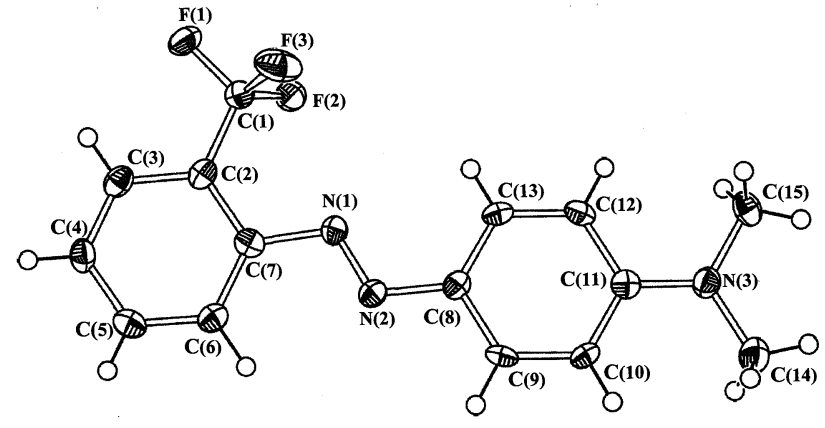

Fig. 2 Molecular structure of 2-TFMDAB with the atom numbering. Thermal ellipsoids of the non-hydrogen atoms are scaled to enclose $50 \%$ probability. The spheres of the hydrogen atoms are drawn in an arbitrary scale.

fractional atomic coordinates and equivalent isotropic thermal parameters for non-hydrogen atoms are given in Table 2 . Selected bond distances, bond angles and torsion angles are listed in Table 3.

The title compound has a trans geometry about the azo linkage. The bond lengths and angles are similar to those of other azobenzene compounds. ${ }^{5-8}$ The $\mathrm{N}=\mathrm{N}$ and the $\mathrm{C}-\mathrm{N}$ bond lengths $[\mathrm{N}(1)-\mathrm{N}(2) \quad 1.271(4) \AA, \quad \mathrm{N}(1)-\mathrm{C}(7) \quad 1.437(4) \AA \quad$ and $\mathrm{N}(2)-\mathrm{C}(8) 1.406(4) \AA]$ are in the normal range of azobenzene compounds. The $\mathrm{N}(1) \cdots \mathrm{F}(2)$ and the $\mathrm{N}(1) \cdots \mathrm{F}(3)$ distances of $2.841(3) \AA$ and $2.956(3) \AA$ are slightly shorter than the sum of the respective van der Waals radii $[1.55 \AA$ for the $\mathrm{N}$ atom and $1.47 \AA$ for the $\mathrm{F}$ atom]. ${ }^{9}$ The two phenyl rings are almost coplanar; the dihedral angle between the planes defined by the two aromatic rings is $5.5(1)^{\circ}$. This molecule adopts a conformation in which $\mathrm{N}(2)$ is oriented anti with respect to carbon $\mathrm{C}(2)$. The corresponding syn conformation would lead to a considerable repulsion between the $\mathrm{CF}_{3}$ group and the electron pair on $\mathrm{N}(2)$, and no doubt a non-planar molecule would result.

An aqueous methanol solution (20\%) of 2-TFMDAB gives a $\lambda_{\max }$ at $440 \mathrm{~nm}\left(\varepsilon_{\max }, 21900\right)$ and $1 \mathrm{M}$ hydrochloric acid solution shows a $\lambda_{\max }$ at $317 \mathrm{~nm}\left(\varepsilon_{\max }, 19970\right)$ with a minor peak at 480 $\mathrm{nm}(\varepsilon, 1560)$. The present $\mathrm{X}$-ray result shows that 2-TFMDAB has a normal trans-azobenzene structure. The abnormal UV-vis absorption spectrum in an acidic solution can be reduced to the existence of the bulky and electronegative $\mathrm{CF}_{3}$ group at the 2position, which prevents protonation at the $\mathrm{N} \beta$ atom of the azo group in an acidic solution, and eventually leads to the formation of a colorless species (III).

\section{References}

1. E. Sawicki, J. Org. Chem., 1957, 22, 621.

2. K. Machida, H. Lee, and A. Kuwae, J. Raman Spectrosc., 1980, 9, 198.

3. H. Lee, K. Machida, A. Kuwae, and Y. Saito, J. Mol. Struct., 1980, 68, 51.

4. H. Lee, K. Machida, A. Kuwae, and I. Harada, J. Raman Spectrosc., 1983, 14, 126.

5. D. Moreiras, J. Solans, X. Solans, C. Miravitlles, G. Germain, and J. P. Declercq, Cryst. Struct. Comm., 1980, 9 , 921.

6. S. A. McIntosh, H. S. Freeman, and P. Singh, Textile Res. J., 1989, 59, 389.

7. A. Whittaker, J. Cryst. Spec. Res., 1992, 22, 151.

8. D.-C. Zhang, L.-Q. Gie, Z.-H. Fei, Y.-Q. Zhang, and K.-B. Yu, Acta Crystallogr., 1998, C54, 1909.

9. A. Bondi, J. Phys. Chem., 1964, 68, 441. 